\title{
Wave propagation in a pre-stressed anisotropic generalized thermoelastic medium
}

\author{
M. D. Sharma \\ Dept. of Mathematics, Kurukshetra University, India-136119 \\ (Received February 21, 2009; Revised October 3, 2009; Accepted December 17, 2009; Online published June 17, 2010)
}

\begin{abstract}
Four attenuated waves propagate in a pre-stressed anisotropic generalized thermoelastic medium. The propagation phenomenon in this medium is explained through two systems. One of them, relating the temperature variation in the medium to the particle displacement, is free from the explicit effect of pre-stress. The other system defines Christoffel equations for the medium. These equations are modified with a matrix, which involves phase direction and pre-stress components. A propagation-attenuation plane is defined for given directions of propagation and attenuation of plane harmonic waves. A finite non-dimensional parameter defines the inhomogeneity strength of an attenuated wave. A complex vector is defined to calculate complex velocities of the four waves from the complex roots of a quartic equation. The complex slowness vector of the attenuated wave in the medium is resolved to calculate its propagation (phase) velocity, quality factor and angle of attenuation. Numerical example is considered to study the propagation characteristics of each of the four attenuated waves in the pre-stressed medium. The presence of anisotropic symmetries and anelasticity are also considered in the medium. Effect of pre-stress is analyzed on the propagation characteristics of each of the four attenuated waves.
\end{abstract}

Key words: Initial stress, thermoelastic, inhomogeneous waves, attenuation.

\section{Introduction}

The crustal rocks are always subjected to stresses. The slow process of creep inside the earth creates a differential stress environment (Hanks and Raleigh, 1980; McGarr, 1980) in the crust, which is responsible for the preferential alignments in the Earth, ranging from mineral orientations, grains, or microcracks to sedimentary folds or regional fractures. The difference between confined tectonic stress and pore-fluid pressure conducts the flow of fluid to a reservoir through the connected cracks. For the presence of pre-stress or aligned cracks, an elastic medium behaves anisotropic to wave propagation. Hence, the almost universal presence of anisotropy is observed in many types of rocks at many depths and in many geological and tectonic environments. Two recent studies (Prikazchikov and Rogerson, 2003; Sharma, 2005) contribute to the understanding of wave propagation characteristics of anisotropic materials under initial stress. A latest book by Carcione (2007) explains the importance of anisotropy for wave propagation studies in real materials.

Temperature variations play a significant role in the modification of cracks and the flow of fluid (Paulsson et al., 1994). These modifications in microcracks are responsible for the dynamism around geothermal reservoirs and the sedimentary basins. Stixrude and Lithgow-Bertelloni (2005) have argued the merit of fundamental thermodynamic relations as the basis for the description of thermoe-

Copyright (c) The Society of Geomagnetism and Earth, Planetary and Space Sciences (SGEPSS); The Seismological Society of Japan; The Volcanological Society of Japan; The Geodetic Society of Japan; The Japanese Society for Planetary Sciences; TERRAPUB.

doi:10.5047/eps.2009.12.004 lastic behaviour of in-situ minerals. Theory of thermoelasticity is used to understand such dynamical systems that involve interactions between mechanical work and thermal changes. Few generalised theories of thermoelasticity have been defined with the introduction of relaxation in temperature field. The theory with one relaxation time (Lord and Shulman, 1967) is termed as LS theory and another with two relaxation times (Green and Lindsay, 1972) is termed as GL theory. Using these modified theories, a large number of problems have been studied on the propagation of plane waves in generalized thermoelastic media (El-Karamany et al., 2002; Sharma et al., 2003). Sharma et al. (2000) studied plane harmonic waves in orthotropic thermoelastic materials. In a recent study (Sharma, 2006), the author considered the general anisotropy in thermoelastic medium and derived a mathematical model to calculate the complex velocities of four waves in the medium. Correspondence was, also, established between the generalised theory of thermoelasticity and the homogenization based $(\mathbf{u}, p)$ theory of poroelasticity.

The attenuation of waves in thermoelastic medium comes from the memory effects allowed to heat conduction. However, a more realistic scheme is defined with memory effects allowed for all the constitutive properties of thermoelastic coupling. Giorgi et al. (2001) studied a linear theory for thermoviscoelastic materials but with thermal behaviour represented through the heat conduction equation. Generalizations of El-Karamany et al. (2002) and El-Karamany and Ezzat (2002) described and established relaxation effects in mechanical properties as well as thermo-mechanical coupling in thermoviscoelastic media. Analogous to the correspondence principle in classical elasticity, the com- 
plex values of appropriate constitutive quantities may define the time-harmonic material dissipation in thermoviscoelastic medium (Caviglia and Morro, 2005).

The present study considers the propagation and attenuation of inhomogeneous waves, in a pre-stressed anisotropic generalized thermoelastic (hereafter, referred as SAGT) medium. In a similar study (Sharma, 2005), the author studied the propagation of homogeneous waves in anisotropic poroelastic medium in the presence of initial stress. But, for the use of Biot's theory of poroelasticity (Biot, 1956) in this paper, the present work can not be obtained through the poroelastic-thermoelastic correspondence (Sharma, 2006). Moreover, the inhomogeneous propagation of attenuated waves is considered in the present work. The complex slowness vector of an inhomogeneous wave is constructed with its propagation direction and inhomogeneity strength. Numerical examples are computed to analyze the propagation characteristics of the attenuated waves in the elastic/viscoelastic media of different anisotropies. Anisotropic variations in propagation characteristics are also analysed for an isotropic medium pre-stressed with hydrostatic pressure.

\section{Definition of the Problem}

The problem is to study the wave propagation in SAGT medium. The attenuated waves of four types can propagate in the medium. The polarisations of these waves may not be along the dynamical axes. The problem may be explained through the following steps.

i) The propagation of plane harmonic waves may be explained through two systems of equations. One of them relates the wave-induced temperature in the medium to the displacement of its particles. The other system provides modified Christoffel equation for the medium. The Christoffel equations are solved into a quartic equation. The four roots of this equation define the complex velocities of the four attenuated waves in SAGT medium.

ii) The inhomogeneity of an attenuated wave in the medium is represented through an inhomogeneity parameter $(\delta)$. For a wave, of given inhomogeneity (value of $\delta$ ), propagating along a given direction $(\hat{\mathbf{n}})$, bisection method is used to calculate a parameter $(\beta)$ to represent homogeneous attenuation. The parameter $\beta$ is used, further, to calculate the phase velocity, quality factor and attenuation angle of the wave considered.

iii) The numerical examples are considered to compute the propagation characteristics (velocity, attenuation) of each of the four inhomogeneous waves in SAGT medium. The presence of anisotropic symmetries and anelasticity in the medium is considered in the numerical models.

\section{SAGT (pre-stressed anisotropic generalized thermoelastic) Medium}

\subsection{Basic equations}

A homogeneous anisotropic thermally conducting elastic solid is considered at a uniform temperature $T_{o}$, in the undisturbed state. In-situ equilibrium is obtained in the presence of homogeneous initial stresses defined by a symmetric tensor $S_{i j}$. Following Biot (1965) the equations for wave motion in this medium, in the absence of body forces and thermal sources, are given by

$$
\begin{aligned}
& \sigma_{i j, j}+S_{j k} \omega_{i k, j}+S_{i k} \omega_{j k, j}-\alpha_{i j}\left(T+\tau_{1}+\delta_{2 m} \dot{T}\right), j=\rho \ddot{u}_{i}, \\
& K_{i j} T_{, i j}-\rho C_{e}\left(\dot{T}+\tau_{o} \ddot{T}\right)=T_{o} \alpha_{i j}\left(\tau_{o} \delta_{1 m} \ddot{u}_{i, j}+\dot{u}_{i, j}\right),
\end{aligned}
$$

where, $K_{i j}$ is (positive-definite) thermal conductivity tensor. The thermoelastic coupling is represented by (nonsingular) tensor $\alpha_{i j} . \quad \rho$ and $C_{e}$ are density and specific heat at constant strain, respectively. The 1 and 2 values of the index $m$ in Kronecker delta $\delta_{j m}$, represent, respectively, the Lord-Shulman (LS) and Green-Lindsay (GL) theories of thermoelasticity. Two thermal relaxation times are given by $\tau_{o} \geq \tau_{1} \geq 0 . u_{i}$ are the components of average displacements of the material particles. $T$ denotes variations in the temperature of the medium in disturbed state. $\omega_{i j}=\left(u_{i, j}-u_{j, i}\right) / 2$ are components of rotation. The incremental stresses $\sigma_{i j}$ in the medium are expressed as

$$
\sigma_{i j}=c_{i j k l} u_{k, l}
$$

where, the fourth rank asymmetric tensor $c_{i j k l}\left(=c_{j i k l}=\right.$ $c_{i j l k}$ ) represents isothermic elastic constants of the medium. Another property, given by

$$
c_{i j k l}-c_{k l i j}=S_{k l} \delta_{i j}-S_{i j} \delta_{k l},
$$

ensures the existence of strain energy density function for the medium (Biot, 1965). All the indices (except $m$ ) can take values of $1,2,3$. Repeated index implies summation. The dot and comma notations denote (partial) differentiation with respect to time and space, respectively.

\subsection{Plane harmonic waves}

For propagation of plane harmonic waves, the unknowns $\left(u_{j}\right.$ and $\left.T\right)$ is written as

$$
\begin{aligned}
u_{j} & =S_{j} \exp \left\{l \omega\left(p_{k} x_{k}-t\right)\right\} ; \quad(j=1,2,3), \\
T & =\Theta \exp \left\{l \omega\left(p_{k} x_{k}-t\right)\right\},
\end{aligned}
$$

where, the components $\left(S_{1}, S_{2}, S_{3}\right)$ define the polarization vector $\mathbf{S}$ for the displacement of the particles in the medium. The vector $\mathbf{p}=\left(p_{1}, p_{2}, p_{3}\right)$ defines slowness of the waves. Analogous to Sharma (2006), the propagation phenomenon of plane harmonic waves in the SAGT medium is explained through two systems. One of them, given by

$$
T=\imath \omega \frac{T_{o}\left(\tau_{0} \delta_{1 m}+\imath / \omega\right)}{K_{r s} p_{r} p_{s}-\rho C_{e}\left(\tau_{0}+\imath / \omega\right)} \alpha_{j k} p_{k} u_{j},
$$

relates the displacement (u) of particles to the temperature change $(T)$ in the medium. 
The other system represents the modified Christoffel equations and is given by

$$
\begin{aligned}
& W_{i k} S_{k}=0 ; \\
& W_{i k}=-\rho \delta_{i k}+c_{i j k l} p_{j} p_{l}+0.5 \Sigma_{i k}-T_{o} \tau^{\prime} \frac{\alpha_{i j} p_{j} \alpha_{k l} p_{l}}{K_{r s} p_{r} p_{s}-\rho C_{e} \tau},
\end{aligned}
$$

where, $\tau=\tau_{0}+\imath / \omega$ and $\tau^{\prime}=\tau_{0} \delta_{1 m}+\tau_{1} \delta_{2 m}+\imath / \omega$ are the complex relaxation times. The matrix $\Sigma$, given by

$$
\Sigma_{i k}=p_{j} p_{k} S_{i j}-p_{i} p_{j} S_{j k}+p_{j} p_{l} S_{j l} \delta_{i k}-p_{j} p_{j} S_{i k},
$$

represents the effect of pre-stress on the wave propagation in SAGT medium. The absence of pre-stress components $S_{i j}$ in relation (6), implies that the pre-stress has no direct effect on wave-induced temperature $T$. However, the effect of pre-stress on the displacement $\left(u_{i}\right)$ results in a change in $T$. A non-trivial solution of the Christoffel equations is ensured by a determinantal equation. Writing slowness $\mathbf{p}=\mathbf{N} / V$, such that row matrix $\mathbf{N}$ satisfies $\mathbf{N N}^{T}=1$, this equation is given by

$$
\begin{aligned}
& \operatorname{det}\left[-\rho h \mathbf{I}+\left(\mathbf{Z}_{N}+0.5 \boldsymbol{\Sigma}_{N}+\mathbf{Y}\right)+\frac{1}{\chi h-1} \mathbf{Y}\right]=0 \\
& h=V^{2}
\end{aligned}
$$

where, $\boldsymbol{\Sigma}_{N}=\mathbf{N S N} \mathbf{N}^{T} \mathbf{I}-\mathbf{S}+\mathbf{S N}^{T} \mathbf{N}-\mathbf{N}^{T} \mathbf{N S}$ and matrix $\mathbf{Z}_{N}=\mathbf{Z}(\mathbf{N}, \mathbf{N})$, as defined in Appendix. For thermal conductivity $\mathbf{K}=K_{o} \mathbf{K}_{\mathbf{a}}$ with anisotropy defined by matrix $\mathbf{K}_{\mathbf{a}}$, and coupling tensor $\alpha=\alpha_{o} \alpha_{\mathbf{a}}$ with anisotropy defined by matrix $\alpha_{\mathbf{a}}$, we have

$$
\begin{aligned}
& \chi=\chi_{o}(1+\imath \eta) / \mathbf{N K}_{\mathbf{a}} \mathbf{N}^{T}, \quad \chi_{o}=\rho C_{e} \tau_{o} / K_{o}, \quad \eta=\left(\omega \tau_{o}\right)^{-1} \\
& \text { and } \\
& \mathbf{Y}=\sigma\left(\delta_{1 m}+\frac{\tau_{1}}{\tau_{o}} \delta_{2 m}+\imath \eta\right)(1+\imath \eta)^{-1} \alpha_{\mathbf{a}} \mathbf{N}^{T} \mathbf{N} \alpha_{\mathbf{a}} \\
& \sigma=T_{o} \alpha_{o}^{2} /\left(\rho C_{e}\right)
\end{aligned}
$$

In Eq. (8), the matrices $\mathbf{Y}$ and $\boldsymbol{\Sigma}_{N}$ represent the effects of thermoelastic coupling and pre-stress, respectively, on the propagation of elastic waves. With the substitution of $\boldsymbol{\Sigma}_{N}=0$, this equation governs the anisotropic propagation of thermoelastic wave (Sharma, 2006).

\subsection{Four attenuated waves}

Following the procedure explained in Sharma (2006), the determinantal Eq. (8) is solved into a quartic equation in $h\left(=V^{2}\right)$. This equation may be written as

$$
h^{4}+C_{1} h^{3}+C_{2} h^{2}+C_{3} h+C_{4}=0 .
$$

The complex coefficients $C_{j}$, for a numerical model of SAGT medium, are functions of phase vector $\mathbf{N}$. Four complex roots (say, $h_{j} ; j=1,2,3,4$ ) of this equation explain the existence and propagation of four attenuated waves in SAGT medium. The four waves are identified with the algebraic expressions of these roots. The attenuated waves corresponding to $h_{1}, h_{2}, h_{3}, h_{4}$ are called the $q P, q S 1, q S 2, q T$ waves, respectively. For any of these four waves, the complex velocity $(V)$ corresponding to the complex vector $\mathbf{N}$ is calculated from the corresponding value of $h$. Thus obtained, slowness vector (i.e., $\mathbf{p}=\mathbf{N} / V$ ) for the wave may be used to calculate its polarization from the system of Eqs. (6).

\subsection{Inhomogeneous plane waves}

In a dissipative medium, an attenuated wave may be homogeneous or inhomogeneous. An angle between propagation vector and attenuation vector, in general, represents the inhomogeneous character of a plane wave. The phase vector $\mathbf{N}$, in real space, yields same direction for propagation vector and attenuation vector and, hence, represents the homogeneous waves. So, an inhomogeneous wave may be represented, only, with $\mathbf{N}$ as a complex (dual) vector.

Consider a plane that contains propagation vector (along, say, $\hat{\mathbf{n}}$ ) and attenuation vector of inhomogeneous plane wave. Let, this plane be formed with two orthogonal unit vectors $\hat{\mathbf{n}}$ and $\hat{\mathbf{m}}$. The complex slowness vector $(\mathbf{p})$ is, then, expressed as

$$
\mathbf{p}=\frac{1}{v}[\hat{\mathbf{n}}+\imath \beta \hat{\mathbf{n}}+\imath \delta \hat{\mathbf{m}}]
$$

where, the non-dimensional parameter $\delta$ measures the inhomogeneity strength of the attenuated wave. A non-zero value of $\delta$, in $(-1,1)$, represents the deviation of inhomogeneous wave from its homogeneous version (i.e., $\delta=0$ ). The coefficient of homogeneous attenuation $(\beta)$ and propagation velocity $(v)$ are the real unknowns, to be determined for given propagation direction $\hat{\mathbf{n}}$, orthogonal direction $\hat{\mathbf{m}}$ and inhomogeneity parameter $\delta$. The expression $\mathbf{p}=\mathbf{N} / V$, such that $\mathbf{N} . \mathbf{N}=1$, of complex slowness vector is used in (10) to yield

$$
\begin{aligned}
& \mathbf{N}=[\hat{\mathbf{n}}(1+\imath \beta)+\imath \delta \hat{\mathbf{m}}] / \sqrt{\left[(1+\imath \beta)^{2}-\delta^{2}\right]} \\
& \frac{v^{2}}{V^{2}}=(1+\imath \beta)^{2}-\delta^{2} .
\end{aligned}
$$

Note that, the phase vector $\mathbf{N}$ is a vector function of only one (real) unknown, i.e., $\beta$. This implies that the complex velocity $V=V(\mathbf{N})$, also, becomes a function of $\beta$, such that $V^{2}=h(\beta)=h_{R}+\imath h_{I}$. The complex value $h=h(\mathbf{N})$, for each of the four waves, is available as one of the roots of quartic equation (9). The second equation in (11), then, written as

$$
v^{2}=\left(1-\delta^{2}-\beta^{2}+2 \imath \beta\right)\left(h_{R}+\imath h_{I}\right),
$$

yields

$$
0=\left(1-\delta^{2}-\beta^{2}\right) h_{I}+2 \beta h_{R}
$$

an equation to be solved for $\beta$ using a bisection method, and

$$
v^{2}=\left(1-\delta^{2}-\beta^{2}\right) h_{R}-2 \beta h_{I},
$$

to calculate $v^{2}$ for $\beta$ obtained from (13). A positive $h_{R}$ and $\beta^{2}<1-\delta^{2}$ ensure a positive value for $v^{2}$. Equation (13) has a root in $\left(0, \sqrt{1-\delta^{2}}\right)$, when $h_{I}<0$. If $h_{I}>0$, then, this root lies in $\left(-\sqrt{1-\delta^{2}}, 0\right)$. This implies that opposite signs of $\beta$ and $h_{I}$ ensures a positive value for $v^{2}$.

The coefficient of homogeneous attenuation $\beta$ is calculated for each of the inhomogeneous waves in SAGT medium. It is used, further, to define quality factor $(Q)$ and 
angle $(\gamma)$ of attenuation, as follows.

$$
Q^{-1}=-\frac{h_{I}}{h_{R}}=\frac{2 \beta}{1-\beta^{2}-\delta^{2}}, \quad \gamma=\cos ^{-1}\left(\frac{\beta}{\sqrt{\beta^{2}+\delta^{2}}}\right) .
$$

\section{Numerical Examples}

Propagation of an attenuated wave is characterised by its phase velocity $(v)$ and quality factor of attenuation $(Q)$. In general, the inhomogeneity of an attenuating wave is represented through the difference in the directions of its propagation vector and attenuation vector. In other words, an angle $(\gamma)$ between equi-amplitude plane and equi-phase plane of an attenuating plane wave represent its inhomogeneous character. To avoid forbidden directions (Krebes and Le, 1994) for $\gamma$, an inhomogeneity of an attenuated wave is defined with the non-dimensional inhomogeneity parameter $(\delta)$. The relation (15) relates the angle $\gamma$ to the parameter $\delta$. Hence, phase velocity $(v)$, quality factor $(Q)$ and attenuation angle $(\gamma)$ are the main propagation characteristics of an inhomogeneous wave. These characteristics are the functions of $\delta$ and, in anisotropic media, these are the functions of propagation direction ( $\hat{\mathbf{n}})$ also. Variations of these characteristics with propagation direction explain the anisotropic behaviour of inhomogeneous waves. The value of $\delta$ defines a general attenuated wave varying from homogenous propagation $(\delta=0)$ to evanescent wave $(\beta=0)$. The arbitrary anisotropy always provides a liberty to consider any realistic anisotropy with symmetries.

The procedures discussed in this study constitute a general mathematical model for propagation of plane harmonic waves in SAGT medium. The use of the derived model in analyzing the propagation characteristics of a realistic thermoelastic medium may be explained through numerical examples. Dolomite rock (Rasolofosaon and Zinszner, 2002) of density $2727 \mathrm{~kg} / \mathrm{m}^{3}$ is considered as SAGT medium and, in two-suffixed notations, its anisotropic elastic constants (in $\mathrm{GPa}$ ) are given by

$$
\begin{aligned}
& c_{11}=106.8 \quad c_{12}=27.1 \quad c_{13}=9.68 \quad c_{14}=-0.03 Z_{1} \\
& c_{15}=0.28 Z_{1} \quad c_{16}=0.12 Z_{2} ; \\
& c_{22}=99.0 \quad c_{23}=18.22 \quad c_{24}=1.49 Z_{1} \quad c_{25}=0.13 Z_{1} \\
& c_{26}=-0.58 Z_{2} ; \\
& c_{33}=54.57 \quad c_{34}=2.44 Z_{1} \quad c_{35}=-1.69 Z_{1} \\
& c_{36}=-0.75 Z_{2} ; \\
& c_{44}=25.97 \quad c_{45}=1.98 Z_{2} \quad c_{46}=0.43 Z_{1} \quad c_{55}=26.05 \\
& c_{56}=1.44 Z_{1} \quad c_{66}=37.82 .
\end{aligned}
$$

The remaining elements of non-symmetric elastic tensor $c_{i j}$ are obtained through the relation defined in Appendix. The amount (in GPa) of initial stress in the medium is assumed through the matrix $\mathbf{S}=$ $\left\{1,0.11 Z_{2}, 0.09 Z_{1} ; 0.11 Z_{2}, 1.1,0.1 Z_{1} ; 0.09 Z_{2}, 0.1 Z_{1}, 0.9\right\}$. A symmetric matrix $\left\{1, .1 Z_{2}, .2 Z_{1} ; .1 Z_{2}, 1.1, .15 Z_{1} ; .2 Z_{1}\right.$, $\left..15 Z_{1}, .9\right\}$ defines the anisotropies of thermal conductivity $\left(\mathbf{K}_{\mathbf{a}}\right)$ as well as of thermoelastic coupling $\left(\alpha_{\mathbf{a}}\right)$. It may be noted that the values $(1,1),(0,1)$ and $(0,0)$ of $\left(Z_{1}, Z_{2}\right)$ represent, respectively, the triclinic, monoclinic and orthotropic anisotropy in the model. Keeping in mind the values for metals, the assumed values for thermal coefficients of SAGT medium are given by $T_{o}=300^{\circ} \mathrm{K}$, $\alpha_{o}=(2.) 10^{-5} c_{11} /{ }^{\circ} \mathrm{K}, C_{e}=400 \mathrm{~J} \mathrm{~kg}^{-1} /{ }^{\circ} \mathrm{K}, K_{o}=400 \mathrm{~W}$ $\mathrm{m}^{-1} /{ }^{\circ} \mathrm{K}$. The relaxation times are assumed as $\tau_{o}=10^{-9}$ sec, $\tau_{1}=0.95 \tau_{o}$.

For general direction $(\theta, \phi)$, in three-dimensional space, the propagation direction $\hat{\mathbf{n}}$ is defined by $(\sin \theta \cos \phi, \quad \sin \theta \sin \phi, \quad \cos \theta)$. The vertical plane $\phi=0.34 \pi$ is the fixed propagation-attenuation plane for numerical computation. In this plane, the propagation direction is considered with $\theta$, varying from 0 to $90^{\circ}$ and the orthogonal vector $\hat{\mathbf{m}}$ is considered along $\theta+\pi / 2$. The three values of $\delta(=0.01,0.1,0.4)$ are used to represent the variations in inhomogeneity strength of a wave. The smallest value $(0.01)$ of $\delta$ represents a nearly homogeneous (or weakly inhomogeneous) wave. It was noted that the thermoelasticity theory (LS or GL) has only a negligible effect on the propagation characteristics of inhomogeneous waves. Hence, the numerical results are exhibited, only, for GL theory (i.e., $m=2$ ). Three main anisotropies (triclinic, monoclinic, orthotropic) are considered in thermoelasticity as well as initial-stress.

Figure 1 exhibits the anisotropic variations of phase velocity $(v)$, quality factor $\left(Q^{-1}\right)$ and attenuation angle $(\gamma)$ for the inhomogeneous thermoelastic $q P$-waves. The velocity plots show that the velocity of $q P$-wave decreases with the increase of inhomogeneity $(\delta)$. This decrease is largest at $\theta=0$ but reduces on shifting away from this direction. The near similarity of velocity plots in three columns of the figure implies that the presence of a reflection symmetry in SAGT medium has a very little effect on the velocity of $q P$-waves. The second row of plots in this figure shows the anisotropic variations of attenuation with the inhomogeneity of wave. It is quite clear from these plots that the attenuation is increasing with the increase of $\delta$. Moreover, the attenuations represented by nearly homogeneous waves (i.e., $\delta=0.01$ ) are negligible as compared to the attenuations from inhomogeneous waves. The effect of monoclinic symmetry is observed only for propagation near $\theta=0$. $Q^{-1}$ is maximum for a value of $\theta$ near $40^{\circ}$. For propagation along $\theta=90^{\circ}$, the effect of wave inhomogeneity is smallest (almost, negligible) on attenuation. The third row of plots in this figure shows the anisotropic variations of angle between directions of propagation and maximum attenuation. The inhomogeneity parameter has a very little effect on this angle. The $q P$-waves are nearly evanescent (i.e., $\gamma=\pi / 2$ ) when propagate along $\theta=0$ and $\theta=90^{\circ}$. The evanescent character of these waves, even when they are nearly homogeneous (i.e., $\delta=0.01$ ), implies that the value of $\beta$ is very small and, hence, a very small contribution of homogeneous waves to the total attenuation. The inhomogeneity angle increases on shifting the propagation away from these directions and gets its maximum around $\theta=40^{\circ}$. The presence of monoclinic symmetry has a significant effect on $\gamma$ but only when $\theta$ is around 0 .

Figure 2 shows the anisotropic variations of the propagation characteristics of $q S 1$-wave in SAGT medium. The velocity $(v)$ decreases with the increase of $\delta$, mainly, near $\theta=0$. The anisotropic symmetries may not affect the magnitude of velocity much but do affect its variations with 

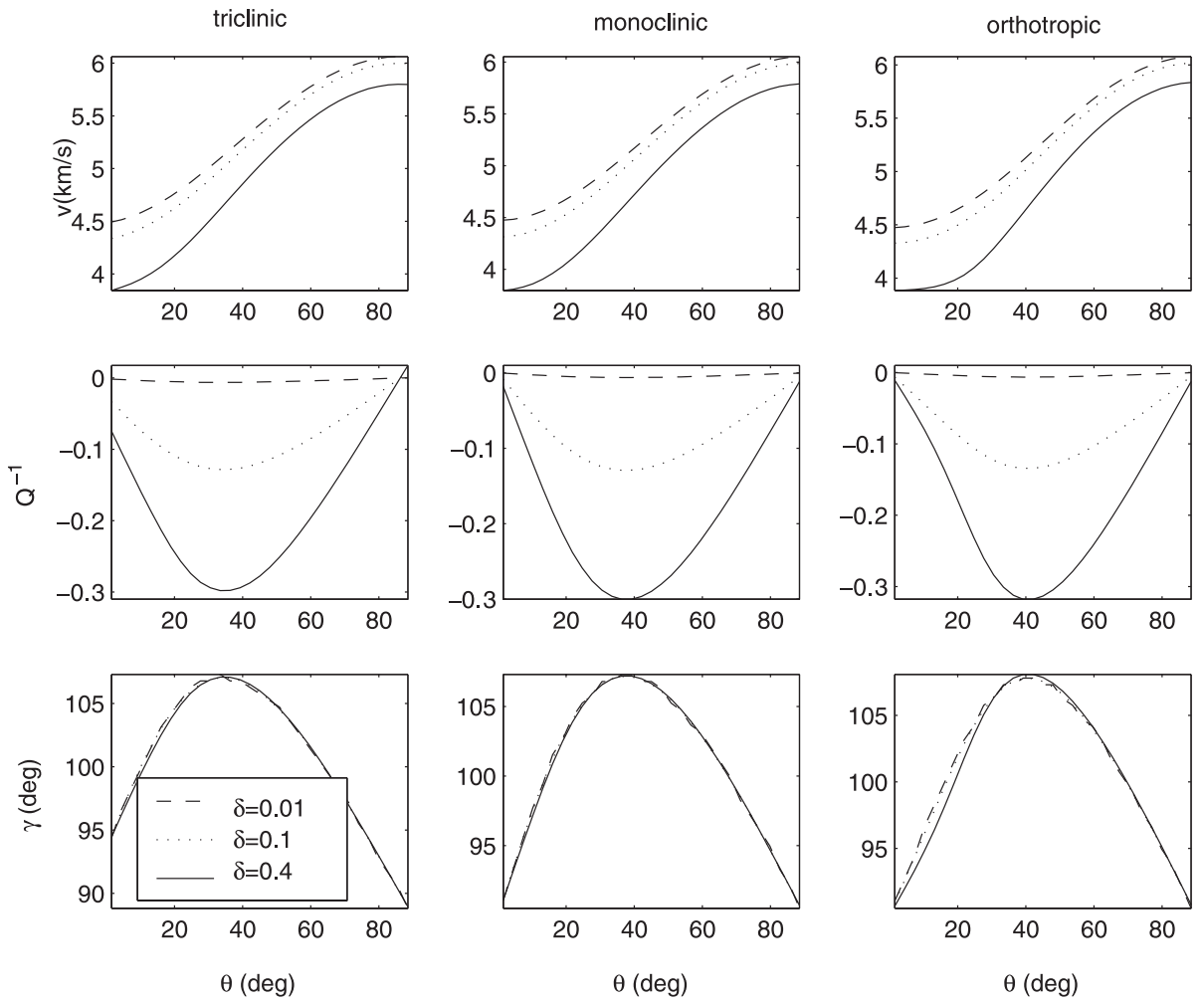

Fig. 1. Variations of propagation velocity $(v)$, quality factor $\left(Q^{-1}\right)$ and attenuation angle $(\gamma)$ of $q P$-wave with propagation direction $(\theta)$ and inhomogeneity parameter $(\delta)$.
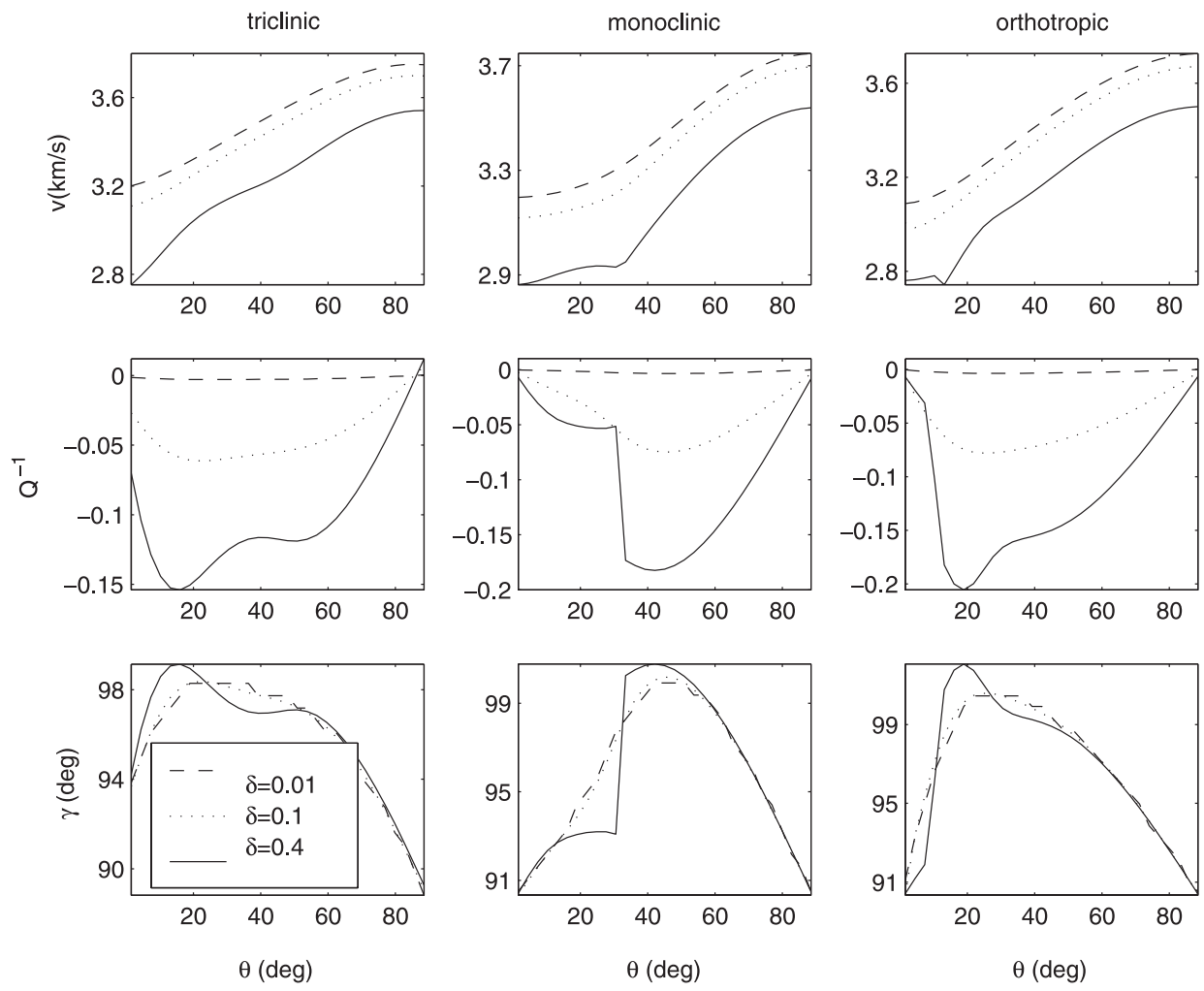

Fig. 2. Same as Fig. 1, but for $q S 1$-wave.

propagation direction. The magnitude of $Q^{-1}$ increases with the increase of $\delta$. For nearly homogeneous (or weakly inhomogeneous) waves (i.e., $\delta=0.01$ ), the value of $Q^{-1}$ is negligible and remain unchanged with the change in propa- gation direction. This implies that contribution of homogeneous waves to attenuation is negligible. Presence of anisotropic symmetry affects the magnitude as well as directional variations of velocity of $q S 1$-wave. The larger 

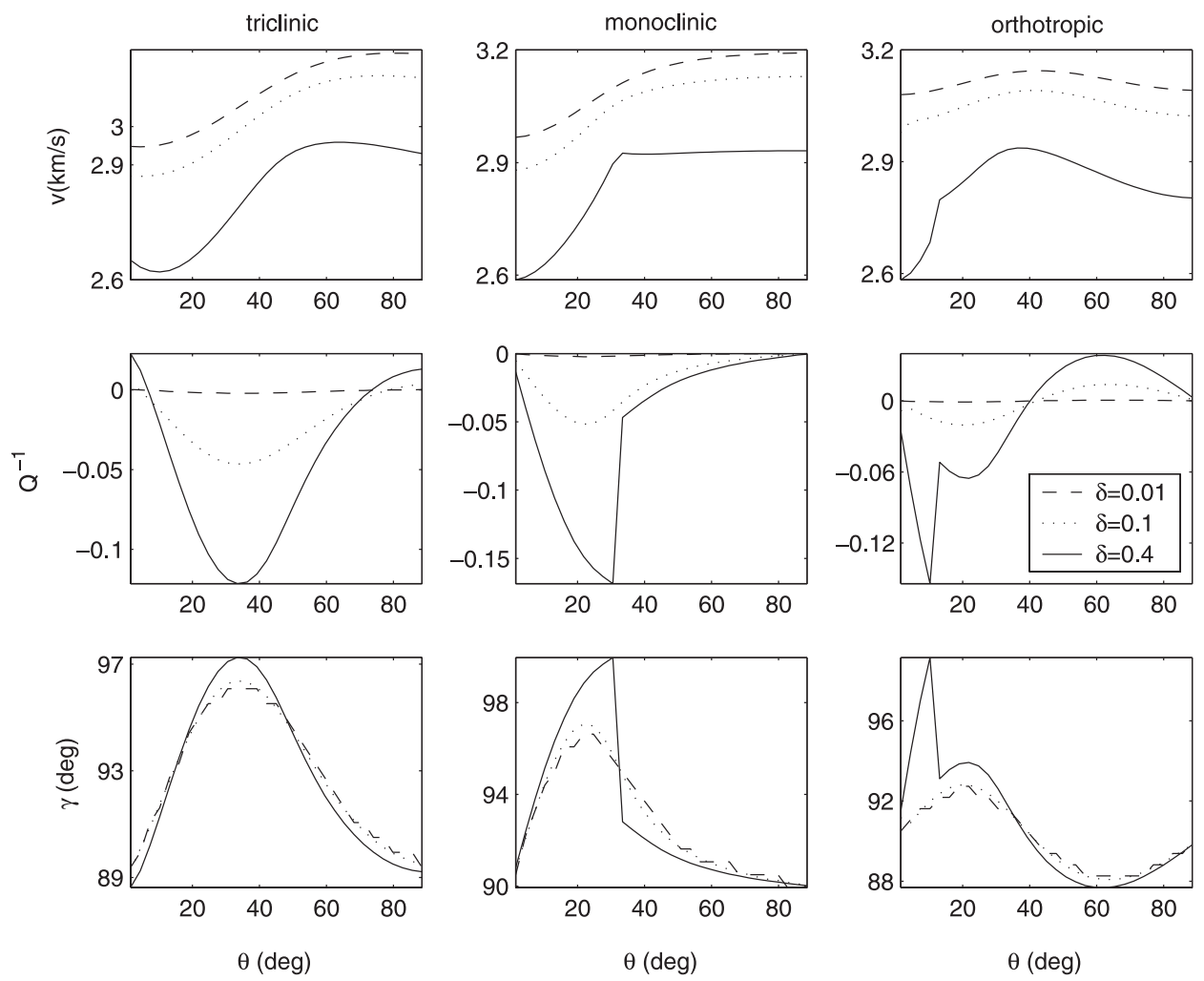

Fig. 3. Same as Fig. 1, but for $q S 2$-wave.
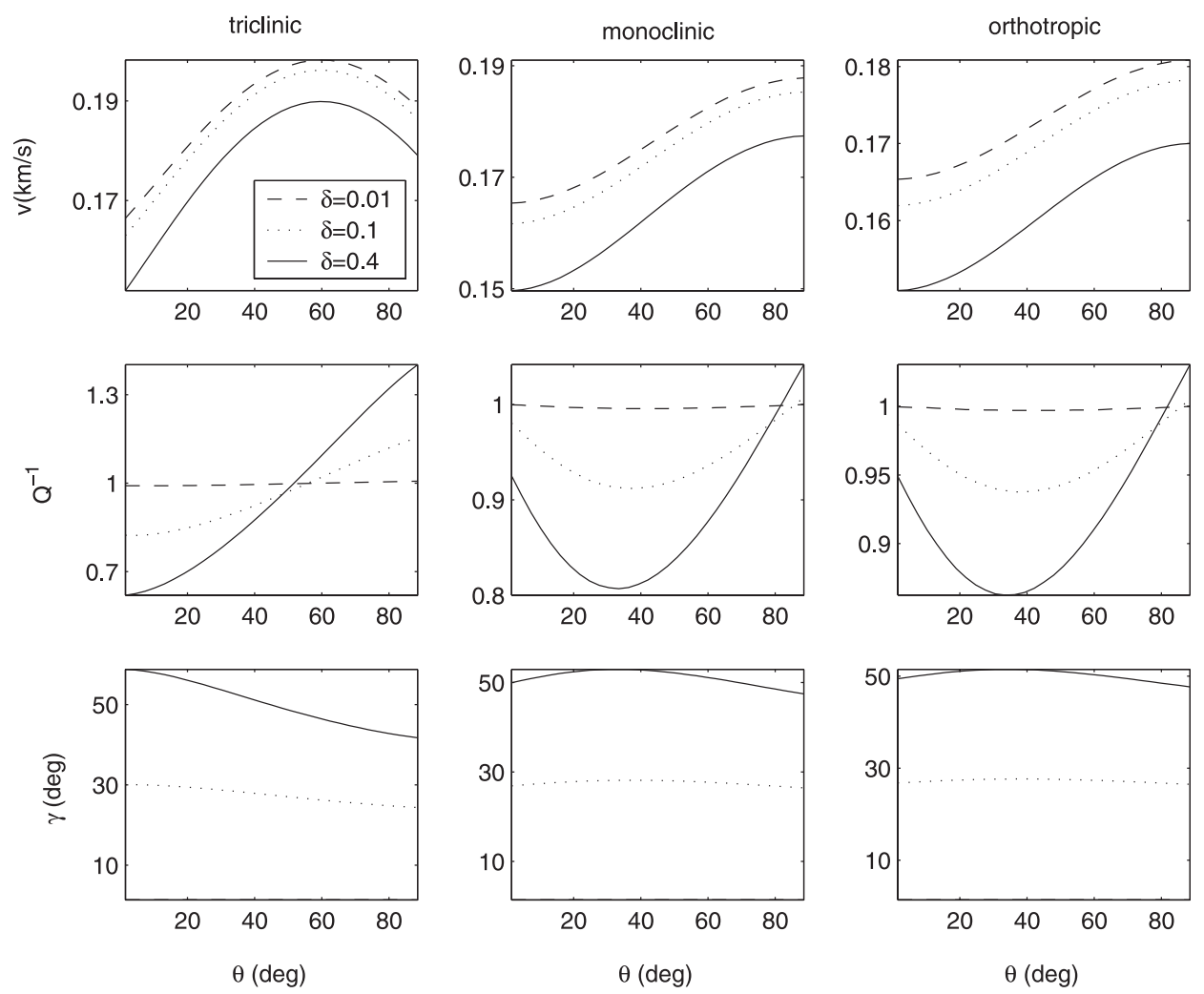

Fig. 4. Same as Fig. 1, but for $q T$-wave.

value of $\delta$ (i.e., 0.4) shows its effect on $\gamma$. The value of $\gamma$ near $90^{\circ}$ implies the near evanescent and strongly inhomogeneous nature of $q S 1$-waves.

The plots in Fig. 3 explains the anisotropic variations of $v, Q^{-1}$ and $\gamma$ for $q S 2$-wave. The effect of inhomogeneity parameter is similar to as observed for $q S 1$-wave. However, the variations with propagation directions are quite different, mainly in the presence of anisotropic symmetries. 

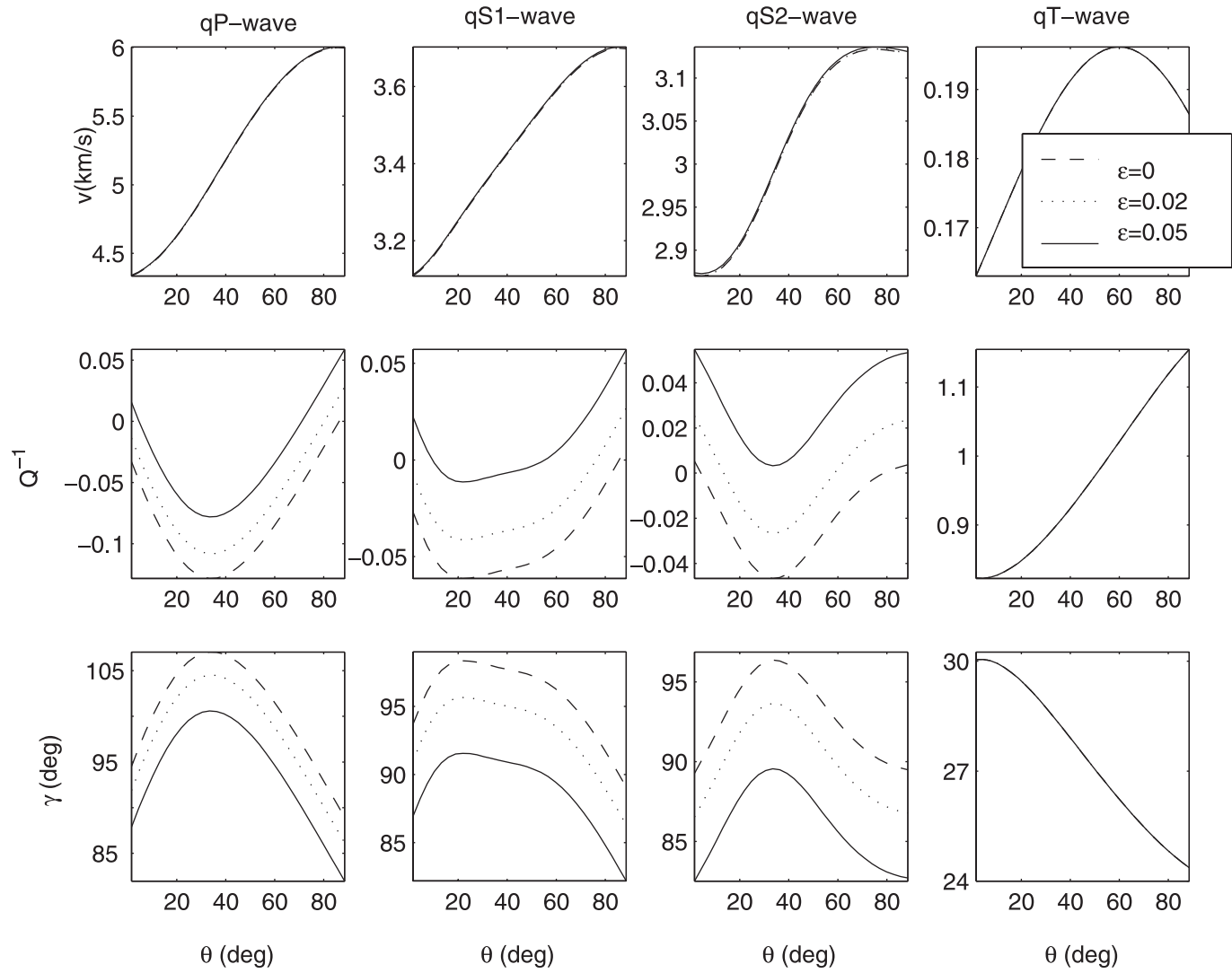

Fig. 5. Variations of propagation velocity $(v)$, quality factor $\left(Q^{-1}\right)$ and attenuation angle $(\gamma)$ with propagation direction $(\theta)$ and anelastic parameter $(\epsilon)$; $\delta=0.2$; anisotropy: triclinic.
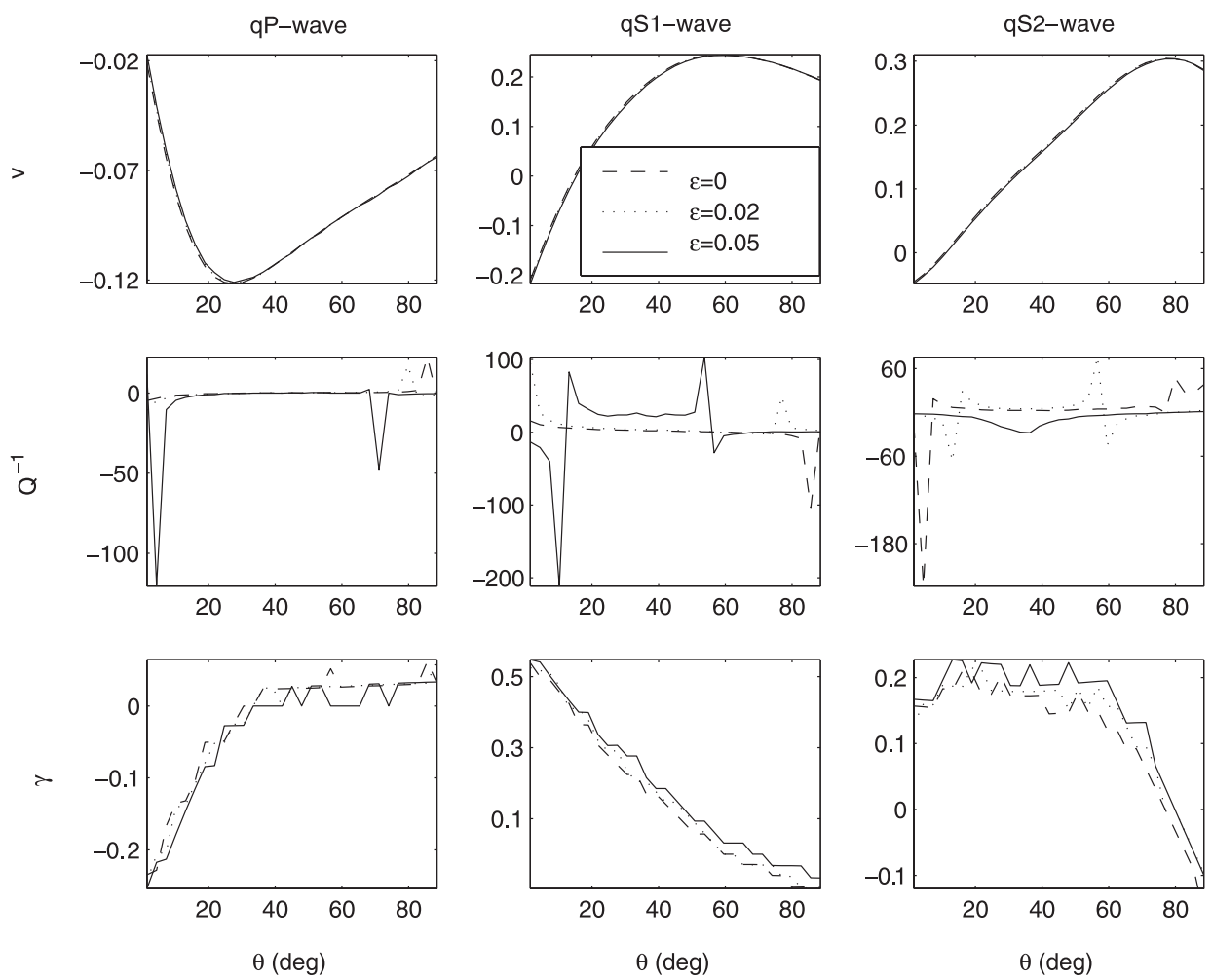

Fig. 6. Change (percent) in propagation velocity $(v)$, quality factor $\left(Q^{-1}\right)$ and attenuation angle $(\gamma)$ due to pre-stress; variations with propagation direction $(\theta)$ and anelastic parameter $(\epsilon) ; \delta=0.2$; anisotropy: triclinic. 

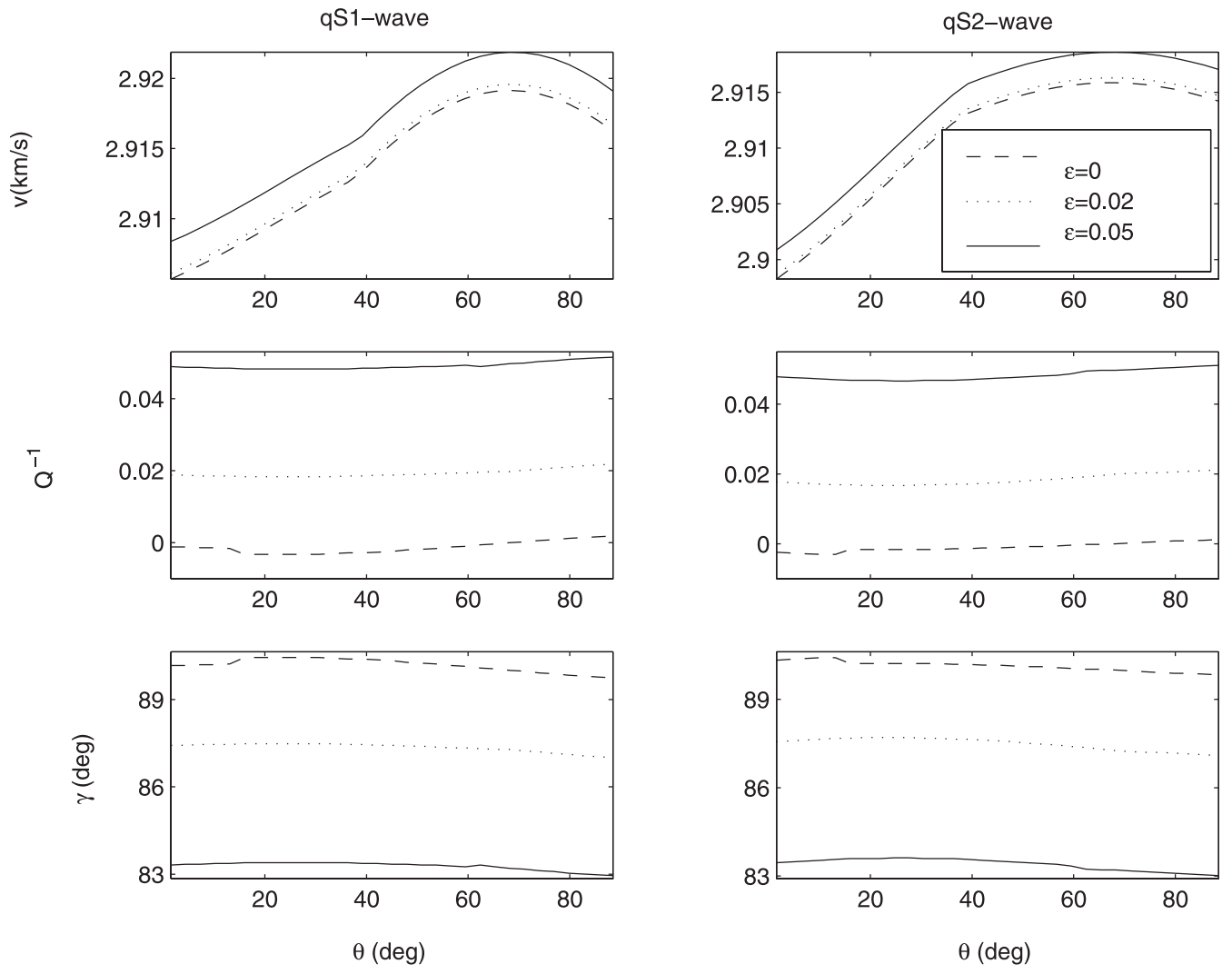

Fig. 7. Variations of propagation velocity $(v)$, quality factor $\left(Q^{-1}\right)$ and attenuation angle $(\gamma)$ in isotropic elastic medium with propagation direction $(\theta)$ and anelastic parameter $(\epsilon) ; \delta=0.2$; pre-stress: hydrostatic.

The anisotropy in the medium has a significant effect on the propagation of $q S 2$-wave. The behavior of anisotropic variations seem to be quite sensitive to the larger (between 0.1 to 0.4 ) values of $\delta$. Similar to $q S 1$-wave, the value of $\gamma$ is around $90^{\circ}$.

Figure 4 shows the propagation characteristics of slowest $(q T)$ wave in the medium, with propagation direction $(\theta)$ and inhomogeneity parameter $(\delta)$. These variations in case of triclinic anisotropy are much different from those for monoclinic and orthotropic anisotropies. The $q T$-wave experience the largest $\left(Q^{-1}\right.$ is around 1) attenuation among all the four waves in the medium. The angle $(\gamma)$ between propagation and attenuation direction is much away from $90^{\circ}$ and varies a lot with the value of $\delta$. For $\delta=0.01$, the zero value of $\gamma$ implies that the $q T$-wave propagates as a homogeneous wave. That means the homogeneous $q T$ waves are contributing much to the attenuation, contrary to the three faster waves in the medium. The attenuation $\left(Q^{-1}\right)$ and inhomogeneity angle $(\gamma)$ of the weakly inhomogeneous waves are not changing with the direction of propagation.

The attenuations exhibited in above figures are due to the thermoelastic character of the medium. The other source of the intrinsic attenuation may be the anelastic nature of the medium. The complex elastic constants for a viscoelastic medium are defined as $c_{i j}(1-\imath \epsilon)$. To check the effect of anelasticity, the propagation characteristics are computed for three values $(0,0.02,0.05)$ of $\epsilon$. The variations of $v, Q^{-1}, \gamma$ of the four waves with propagation direction are exhibited in Fig. 5. The waves are inhomogeneous $(\delta=$ 0.2 ) and medium is general anisotropic (i.e., triclinic). The first row of plots in this figure implies that viscoelasticity in the medium may not have any effect on the phase velocities of any of the four waves. However, attenuation angles $\gamma$ and $Q^{-1}$ of three faster waves are increasing with $\epsilon$. The only exception is $q T$-wave, which is not affected by the anelastic nature of the medium.

The plots in Fig. 6 present the percent change in velocity $(v)$, attenuation amount $\left(Q^{-1}\right)$ and attenuation angle $(\gamma)$ due to the presence of pre-stress. Three values of $\epsilon=0,0.02,0.05$ represent the variations in the anelastic character of the medium. Numerical results show that the slowest $(q T)$ wave is unaffected by the presence or absence of pre-stress. Hence, in Fig. 6, the results are exhibited, only, for three faster waves. From the first row of plots it is noted that effect of pre-stress is more on slower waves. However, the change is not more than 0.3 percent and hence may not be significant. The plots in the second row in the figure imply that the effect of pre-stress is quite large on the attenuation but only for propagation along some particular directions. The effect of pre-stress on $Q$ may change with the viscoelastic character of the medium. Similar to velocities, the effect of pre-stress on attenuation angle may not be considered significant.

The anisotropy in the medium is of two types. One is inherent elastic anisotropy and other is due to the presence of pre-stress. The stress-induced anisotropy may, also, be observed in an isotropic elastic medium. So, the computations are carried out to calculate the propagation characteristics of attenuated waves in an isotropic thermoelastic medium (with non-zero elastic constants $c_{11}=c_{22}=c_{33}=110$ 
$\mathrm{GPa}, c_{44}=c_{55}=c_{66}=24 \mathrm{GPa}, c_{12}=c_{21}=c_{13}=c_{31}=$ $\left.c_{23}=c_{32}=c_{11}-2 c_{66}\right)$ in the presence hydrostatic prestress $(\mathbf{S}=\mathbf{I} \mathrm{GPa})$. All the waves are inhomogeneous with $\delta=0.2$. It is calculated that $q P$-wave and $q T$-wave behave isotropic with constant values of $\left(v, Q^{-1}, \gamma\right)$, given by $\left(6.224 \mathrm{~km} / \mathrm{s}, 0.02,87^{\circ}\right)$ and $\left(0.1713 \mathrm{~km} / \mathrm{s}, 1,26.5^{\circ}\right)$, respectively. However, the effect of stress-induced anisotropy is observed on two split-shear $(q S 1, q S 2)$ waves. Figure 7 exhibits, the anisotropic variations of $v, Q^{-1}$ and $\gamma$ for $q S 1$ and $q S 2$ waves. The anisotropy induced by hydrostatic prestress is affecting mainly the velocities. The presence of anelasticity does not show much effect on this anisotropic behavior.

\section{Concluding Remarks}

The numerical results in previous section are computed only for some particular numerical models of SAGT medium. Hence, the analysis of these results may not qualify for generalization. However, the few noticeable points may be explained as follows.

i) The slower is an elastic wave, more it is affected by the presence of anisotropic symmetry. The presence of anisotropic symmetries have a very significant effect on the variations of velocity with propagation direction. The negligible effect of anisotropic symmetry on the velocities of $q P$ and $q T$ waves may indicate that the quasi-longitudinal waves are less sensitive to the presence of anisotropic symmetry.

ii) The attenuations represented by weakly inhomogeneous (i.e., $\delta=0.01$ ) faster waves are negligible. This implies that the large attenuation in a dissipative medium may be explained only with strongly inhomogeneous waves. The values of $\gamma$ near $90^{\circ}$ imply the near-evanescent character of inhomogeneous waves in the dissipative medium.

iii) The $q T$-waves experience the largest attenuation among all the four waves in the medium. The angle $(\gamma)$ between propagation and attenuation direction of $q T$-wave is much away from $90^{\circ}$ and varies a lot with the value of $\delta$. For $\delta=0.01$, the significant value of $Q^{-1}$ and zero value of $\gamma$ implies that attenuated $q T$ wave propagates as a homogeneous wave. That means, homogeneous $q T$-waves may be able to explain large attenuation also, contrary to the three faster waves in the medium.

iv) Viscoelasticity in the medium may not have any effect on the phase velocities of any of the four waves. However, the attenuation angles $(\gamma)$ and coefficients $\left(Q^{-1}\right)$ of three faster waves increase with $\epsilon$. The exception is $q T$-wave, which is not affected by the anelastic nature of the medium.

v) The propagation of quasi-thermal $(q T)$ wave may not be affected with the presence of pre-stress in SAGT medium. The other three waves may not be changing their velocities and attenuation angle with the presence of pre-stress. However, pre-stress may affect the attenuation of these waves but only for few propagation directions. This implies that, to affect the velocities of the waves, the pre-stress values should be much greater than that assumed in the numerical model.

vi) The effect of anisotropy induced by hydrostatic prestress in an isotropic thermoelastic medium is observed only on two split-shear $(q S 1, q S 2)$ waves. The isotropic propagation behavior of other two $(q P$, $q T$ ) waves is not affected by the presence this stressinduced anisotropy.

The work presented relates the geophysical properties (anisotropy, initial-stress, thermoelasticity) of a realistic medium to its propagation characteristics (velocities, attenuation, polarizations, phase shift, reflection coefficients). For the presence of anelastic attenuation and anisotropic symmetries, these relations may provide improved constraints on thermal/compositional structure and deformational processes in the interior of Earth. When supported with a real data, these constraints may be used to understand a variety of geophysical problems. Few relevant problems are explained as follows.

a) Seismic waves generated in Earth's interior provide images that help us to better understand the pattern of mantle convection that drives plate motions. Anisotropy and dissipation, which also influence seismic-wave propagation, may be characterized better to extract additional information on flow directions, temperature variations and the presence of partial melting (Romanowicz, 2008).

b) The boundary between the core and mantle is one of the most enigmatic regions of Earth's interior. It holds the key to understand a host of geophysical phenomena-including the formation of plumes in the mantle, interactions between core and mantle, and the ultimate fate of subducting slabs of crust that are driven into the interior by tectonic forces. Investigations of this region largely depend on interpreting the behaviour of seismic waves, which have shown that it is highly complex (Duffy, 2004)

c) $\mathrm{The} \mathrm{MgSiO}_{3}$ perovskite, generally accepted to be the major component of the lower mantle. It is found to be highly anisotropic in all portions of the lower mantle and the nature of anisotropy changes significantly with depth. Wentzcovitch et al. (1998) calculated anisotropy of seismic wave velocities as a function of pressure (depth). Anisotropy at the topmost lower mantle can be attributed to the preferred orientation of perovskite.

d) Knowledge of the elastic properties of the dominant $(\mathrm{Mg}, \mathrm{Fe}, \mathrm{Al})(\mathrm{Si}, \mathrm{Al}) \mathrm{O}_{3}$ perovskite phase of the Earth's lower mantle, including the pressure and temperature dependence of the bulk and shear moduli, is critical for analyses of its chemical composition and thermal regime including the significance of the lateral variations of seismic wave speeds (Jackson, 1998; Deschamps and Trampert, 2004; Mattern et al., 2005).

e) The prediction of pre-drill overpressure is required for the monitoring of hydrocarbon production in the boreholes (Sayers et al., 2002).

f) The extent of fracturing in a region of a borehole, which is a vital factor in the extraction of oil and geothermal heat. The information on fracture distribu- 
tion (from velocity inversion) is used to estimate the anisotropic permeability of the fracture rock system (Gibson and Toksoz, 1990).

\section{Appendix A.}

A non-symmetric square matrix of order six, $\left\{c_{i j}\right\}$, is used to denote the elastic properties of the medium in twosuffix notations. In the presence of initial stress $\left(S_{i j}\right)$ in the medium, the asymmetry of the elastic tensor $\left(c_{i j}\right)$ is expressed through the relations

$$
\begin{aligned}
& c_{12}-c_{21}=S_{22}-S_{11}, \quad c_{13}-c_{31}=S_{33}-S_{11}, \\
& c_{23}-c_{32}=S_{33}-S_{22} ; \\
& c_{14}-c_{41}=c_{24}-c_{42}=c_{34}-c_{43}=S_{23}, \\
& c_{15}-c_{51}=c_{25}-c_{52}=c_{35}-c_{53}=S_{13}, \\
& c_{16}-c_{61}=c_{26}-c_{62}=c_{36}-c_{63}=S_{12}, \quad c_{54}=c_{45}, \\
& c_{64}=c_{46}, \quad c_{65}=c_{56} .
\end{aligned}
$$

Consider two row matrices $\mathbf{U}$ and $\mathbf{V}$ of three elements each. These matrices define the elements of a square matrix $\mathbf{Z}(\mathbf{U}, \mathbf{V})$ of order three, as follows.

$$
\begin{aligned}
& Z_{11}=\mathbf{U A V}^{T}, \quad Z_{22}=\mathbf{U B V} \mathbf{V}^{T}, \quad Z_{33}=\mathbf{U} \mathbf{C V}^{T}, \\
& Z_{12}=\mathbf{U D V}^{T}, \quad Z_{13}=\mathbf{U E V}^{T}, \quad Z_{21}=\mathbf{U} \mathbf{F} V^{T}, \\
& Z_{23}=\mathbf{U G V}^{T}, \quad Z_{31}=\mathbf{U} \mathbf{H} \mathbf{V}^{T}, \quad Z_{32}=\mathbf{U} \mathbf{J V}^{T},
\end{aligned}
$$

where $\mathbf{V}^{T}$ is the transpose of $\mathbf{V}$ and

$$
\begin{aligned}
& \mathbf{A}=\left\{c_{11}, c_{16}, c_{15} ; c_{61}, c_{66}, c_{65} ; c_{51}, c_{56}, c_{55}\right\} ; \\
& \mathbf{B}=\left\{c_{66}, c_{62}, c_{64} ; c_{26}, c_{22}, c_{24} ; c_{46}, c_{42}, c_{44}\right\} ; \\
& \mathbf{C}=\left\{c_{55}, c_{54}, c_{53} ; c_{45}, c_{44}, c_{43} ; c_{35}, c_{34}, c_{33}\right\} ; \\
& \mathbf{D}=\left\{c_{16}, c_{12}, c_{14} ; c_{66}, c_{62}, c_{64} ; c_{56}, c_{52}, c_{54}\right\} ; \\
& \mathbf{E}=\left\{c_{15}, c_{14}, c_{13} ; c_{65}, c_{64}, c_{63} ; c_{55}, c_{54}, c_{53}\right\} ; \\
& \mathbf{F}=\left\{c_{61}, c_{66}, c_{65} ; c_{21}, c_{26}, c_{25} ; c_{41}, c_{46}, c_{45}\right\} ; \\
& \mathbf{G}=\left\{c_{65}, c_{64}, c_{63} ; c_{25}, c_{24}, c_{23} ; c_{45}, c_{44}, c_{43}\right\} ; \mathbf{H}=\left\{c_{51}, c_{56}, c_{55} ; c_{41}, c_{46}, c_{45} ; c_{31}, c_{36}, c_{35}\right\} ; \\
& \mathbf{J}=\left\{c_{56}, c_{52}, c_{54} ; c_{46}, c_{42}, c_{44} ; c_{36}, c_{32}, c_{34}\right\},
\end{aligned}
$$

are square matrices of order three.

\section{References}

Biot, M. A., The theory of propagation of elastic waves in a fluid-saturated porous solid, I. Low-frequency range, II. Higher frequency range, $J$. Acoust. Soc. Am., 28, 168-191, 1956.

Biot, M. A., Mechanics of Incremental Deformation, John Wiley, New York, 1965.

Carcione, J. M., Wave Fields in Real Media: Wave Propagation in Anisotropic Anelastic, Porous and Electromagnetic Media, Elsevier, Amsterdam, The Netherlands, 2007.

Caviglia, G. and A. Morro, Harmonic waves in thermoviscoelastic solids, Int. J. Engng. Sci., 43, 1323-1336, 2005.
Deschamps, F. and J. Trampert, Towards a lower mantle reference temperature and composition, Earth Planet. Sci. Lett., 222, 161-175, 2004.

Duffy, T. S., Earth science: Deeper understanding, Nature, 430, 409-410, 2004.

El-Karamany, A. S. and M. A. Ezzat, The uniqueness and reciprocity theorems for generalised thermo-viscoelasticity with two relaxation times, Int. J. Engng. Sci., 40, 1275-1284, 2002.

El-Karamany, A. S., M. A. Ezzat, and M. I. Othman, State-space approach to two-dimensional generalised thermo-viscoelasticity with two relaxation times, Int. J. Engng. Sci., 40, 1251-1274, 2002.

Gibson, Jr. R. L. and M. N. Toksoz, Permeability estimation from velocity anisotropy in fractured rocks, J. Geophys. Res. 95, 15643-15656, 1990.

Giorgi, C., M. G. Naso, and E. Vuk, Exponential stability in viscoelastic and elastic systems with thermal memory, Int. J. Differ. Eqs. Appl., 2, 55-91, 2001.

Green, A. E. and K. A. Lindsay, Thermoelasticity, J. Elasticity, 2, 1-7, 1972

Hanks, B. C. and C. B. Raleigh, The conference on magnitude of deviatoric stresses in the earth's crust and uppermost mantle, J. Geophys. Res., $\mathbf{8 5}$, 6083-6085, 1980.

Jackson, I., Elasticity, composition and temperature of the Earth's lower mantle: a reappraisal, Geophys. J. Int., 134, 291-311, 1998.

Krebes, E. S. and L. H. T. Le, Inhomogeneous plane waves and cylindrical waves in anisotropic anelastic media, J. Geophys. Res., 99, 2389923919, 1994

Lord, H. W. and Y. Shulman, The generalized dynamic theory of thermoelasticity, J. Mech. Phys. Solids, 15, 299-309, 1967.

Mattern, E., J. Matas, Y. Ricard, and J. Bass, Lower mantle composition and temperature from mineral physics and thermodynamic modeling, Geophys. J. Int., 160, 973-990, 2005.

McGarr, A., Some constraints on levels of shear stress in the crust from observation and theory, J. Geophys. Res., 85, 6231-6238, 1980.

Paulsson, B. N. P., J. A. Meredith, Z. Wang, and J. W. Fairborn, The Steepbank crosswell seismic project: Reservoir definition and evaluation of steamflood technology in Alberta tar sands, The Leading Edge, 13, 737 747, 1994.

Prikazchikov, D. A. and G. A. Rogerson, Some comments on the dynamic properties of anisotropic and strongly anisotropic pre-stressed elastic solids, Int. J. Engng. Sci., 41, 149-171, 2003.

Rasolofosaon, P. N. J. and B. E. Zinszner, Comparison between permeability anisotropy and elasticity anisotropy of reservoir rocks, Geophysics, 67, 230-240, 2002.

Romanowicz, B., Using seismic waves to image Earth's internal structure, Nature, 451, 266-268, 2008.

Sayers, C. M., G. M. Johnson, and G. Denyer, Predrill pore-pressure prediction using seismic data, Geophysics, 67, 1286-1292, 2002.

Sharma, M. D., Effect of initial stress on the propagation of plane waves in a general anisotropic poroelastic medium, J. Geophys. Res., 110, B11307, 2005.

Sharma, M. D., Wave propagation in anisotropic generalized thermoelastic medium, J. Thermal Stresses, 29, 629-642, 2006.

Sharma, J. N., V. Kumar, and S. P. Sud, Plane harmonic waves in orthotropic thermoelastic materials, J. Acoust. Soc. Am., 107, 293-305, 2000.

Sharma, J. N., V. Kumar, and D. Chand, Reflection of generalized thermoelastic waves from the boundary of a half-space, J. Thermal Stresses, $\mathbf{2 6}$, 925-942, 2003.

Stixrude, L. and C. Lithgow-Bertelloni, Thermodynamics of mantle minerals-I. Physical properties, Geophys. J. Int., 162, 610-632, 2005.

Wentzcovitch, R. M., B. B. Karki, S. Karato, and C. R. S. Da Silva, High pressure elastic anisotropy of $\mathrm{MgSiO} 3$ perovskite and geophysical implications, Earth Planet. Sci. Lett., 164, 371-378, 1998.

M. D. Sharma (e-mail: mohan_here@rediffmail.com) 